\title{
The simulations of the powered resonance tube driven by annular jet
}

\author{
Tian Zhangfu \\ Institute of Optical-electronic Science and Engineering, \\ National University of Defense Technology \\ Changsha, People's Republic of China, 410073 \\ e-mail:kdtzf103@sohu.com \\ Gong Changchao \\ Institute of Optical-electronic Science and Engineering, \\ National University of Defense Technology \\ Changsha, People's Republic of China, 410073 \\ e-mail: gccnudt@163.com
}

\begin{abstract}
In order to generate low frequency acoustic wave and minish the ratio of the length to diameter, it is appropriate to instead the circular jet by annular jet as the high pressure gas power. So the larger diameter of jet and resonator are got in the condition of the same pressure and flux. Flow simulations have been performed in order to better understand the behavior of the powered resonance tube (PRT) driven by annular. Simulation results show the PRT can be excited by a high-speed annular jet, and produce low frequency high-amplitude dynamic pressures and acoustic emission. The compression wave and expansion wave generate and transmit into the resonance tube alternately in a cycle. So a cycle can be decomposed to two distinct stages of filling and evacuation. Pneumatic process in each cycle generally can be divided into four steps: compression wave introducing, Compression wave reflecting, expansion wave introducing, expansion wave reflecting. The two Compression wave phases are the filling process and the . two expansion wave phases are the evacuation process.
\end{abstract}

Keywords-PRT; simulation; aeroacoustic; low frequency; resonance

\section{INTRODUCTION}

Hartmann discovered the resonance tube phenomenon in 1918 . He demonstrated the possibility of obtaining high acoustic efficiencies and numerous other studies were done as acoustic generators ${ }^{[1 \sim 3]}$, ignitors ${ }^{[4,5]}$ or active flow control actuators ${ }^{[6 \sim 10]}$.

The PRT is a static device based on aeroacoustic principles and consists of an open-closed quarterwavelength tube resonator, excited by a high-speed jet that impinges normally on the open end of the tube. The PRT is an effective device for producing high-amplitude dynamic pressures and acoustic emission over a wide range of frequencies (i.e., around $10 \mathrm{~Hz}-25 \mathrm{kHz}$ ) at very high amplitude (i.e., 150+ dB).

In some applications, relatively low frequencies is required. The resonance tube length is inversely proportional to the resonant frequency with the use of a quarter-wavelength resonance tube, and is therefore quite

\author{
Sun Haiyang \\ Institute of Optical-electronic Science and Engineering, \\ National University of Defense Technology \\ Changsha, People's Republic of China, 410073 \\ e-mail: sunhy@nudt.edu.cn \\ Kong Xiaopeng \\ Institute of Optical-electronic Science and Engineering, \\ National University of Defense Technology \\ Changsha, People's Republic of China, 410073 \\ e-mail: kongxp@nudt.edu.cn
}

long for the case of low resonant frequencies. Oscillation is becoming difficult, for the length of resonator is more than 30 times of the diameter. For example, if we consider a resonant frequency $\mathrm{f}=50 \mathrm{~Hz}$ in air at standard temperature, the quarter-wave length is about $1.7 \mathrm{~m}$.

When the gas supply constraints may be incompatible with the circular jet, it is impossible using the large diameter to minish the ratio of the length to diameter. It is possible way instead circular jet by annular jet, as the same pressure and flux, the large diameter jet and resonator are got, and then the ratio of the length to diameter can be minimize to acceptable.

In the following section, we focus on simulations of the powered resonance tube driven by annular jet. We discuss our progress in the flow field simulation of PRT.

\section{CONTROL EQUATIONS}

The numerical simulation of PRT based on compressible Navier-Stokes flow equations. For twodimensional Euler equations in cylindrical coordinates, the conservation of continuity, momentum and energy equations take the vector form

$$
\frac{\partial \mathbf{U}}{\partial t}+\frac{\partial \mathbf{F}}{\partial x}+\frac{\partial \mathbf{G}}{\partial r}+\mathbf{H}=0
$$

As the column vector $\mathbf{U}, \mathbf{F}, \mathbf{G}, \mathbf{H}$ take the form

$$
\mathbf{U}=\left[\begin{array}{c}
\rho \\
\rho u \\
\rho v \\
e
\end{array}\right] \mathbf{F}=\left[\begin{array}{c}
\rho u \\
\rho u^{2}+p \\
\rho u v \\
(e+p) u
\end{array}\right]
$$




$$
\mathbf{G}=\left[\begin{array}{c}
\rho v \\
\rho u v \\
\rho v^{2}+p \\
(e+p) v
\end{array}\right] \mathbf{H}=\frac{1}{r}\left[\begin{array}{c}
\rho v \\
\rho u v \\
\rho v^{2} \\
(e+p) v
\end{array}\right]
$$

Among them

$$
e=p /(\gamma-1)+(\rho / 2)\left(u^{2}+v^{2}\right)
$$

Type (5.1) (5.3), $t, x, r$ is respectively for the time, the axial and radial coordinate; $\rho, p, u, v, e, \gamma$ is respectively the density, pressure, axial component of velocity, the radial component of velocity, total energy and specific heat ratio of the gas.

For the flow oscillation in the resonance tube, calculation of time step is very small to reflect the characteristics of the flow, and it is convenient using the explicit format. For suppressing the numerical oscillation, artificial viscous is used which is the small amount and will not affect the calculation accuracy.

\section{FLOW SIMULATION MODEL OF PRT}

The PRT generally consists of two parts, annular nozzle and the resonant tube. Nozzle is to provide high pressure and speed annular gas jet. The jet flows directly into the resonance cavity in the inner tube. The jet is resonant with the gas in the resonance cavity. The gas is swallowed and spitted out by the resonance cavity, and then the high pressure disturbance and the high-intensity sound waves is produced out of the resonant tube. The schematic structure of the PRT is shown in fig .1. Among them, the nozzle diameter $\mathrm{d} 2$ is $34 \mathrm{~mm}$, the diameter of plug in the nozzle center $\mathrm{d} 1$ is $28 \mathrm{~mm}$,. The resonance cavity diameter $\mathrm{D}=40 \mathrm{~mm}$, the length of tube 1 is $150 \mathrm{~mm}$, injection spacing $\mathrm{H}$ is $50 \mathrm{~mm}$, and injection pressure is 10 atm.

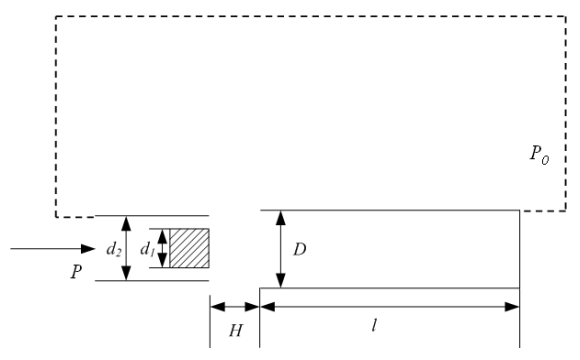

Figure 1. Schematic structure of PRT

\section{THE RESULT OF FLOW SIMULATION OF PRT}

The resonant process of static pressure and velocity distribution are shown in fig .2 and fig .3. Static pressure and Mach number distribution in axis of the resonance tube is s shown in fig.4. In these figures, (a) (c) are the evacuation process, $(\mathrm{d}) \sim(\mathrm{g})$ the intake process, $(\mathrm{h})$ is the beginning of the next cycle. It is worth notice that propagation path of the compression wave and expansion wave includes injection spacing, and this can change the frequency.

The compression wave and expansion wave generate and transmit into the resonance tube alternately in a cycle. So a cycle can be decomposed to two distinct stages of filling and evacuation. In the filling stage, compression wave is introduced to the resonance cavity, and reflected in the bottom of the resonance tube, the pressure in the resonance tube increases gradually; In the evacuation stage, the compression wave is introduced to the free field and expansion wave generates in the open end of resonance tube, expansion wave is introduced to the resonance cavity, and reflected in the bottom of the resonance tube, the pressure in the resonance tube reduces gradually. When the pressure in the cavity is smaller than the outside pressure, gas and the compression wave being back into the cavity, the next cycle begins.

Pressure distribution within the same cross section in the cavity is uniform, and pressure disturbance just appears in the injection of the open end of resonance tube. This is caused by different flow velocity between the area close to wall and the area near axis.

Velocity distribution is more complex than the pressure distribution and the structure of flow field changes over all the time. In the filling stage, it exists backflow in the resonance tube, as shown in fig .3(e). The jet parallels to the axis, flows into the resonance tube near the tube wall, and backflow is out the tube along axis of the tube. This leads the pressure in the resonance cavity to increase uniformly. In the evacuation stage, the jet deflexes away from the resonance tube tip.

The pressure on the axis does not appear discontinuity, and it obviously is no shock wave formed but compression wave. It is different with the traditional approach driven by circular jet, which there is a shock out the mouth of the tube ${ }^{[3]}$. Maximum pressure in the resonance tube is 3.3atm, occurred after the reflected compression wave. Minimum pressure is $0.6 \mathrm{~atm}$, occurred after reflection expansion wave. The resonance cavity forms negative pressure zone and the pressure in the tube is lower than that of the environment. The velocity along the axis is subsonic, and only the velocity near the nozzle in the evacuation process has reached the speed of sound.

As the fig .4 shows, the compression wave and expansion wave are generated near the mouth of tube, and spread into and out of the tube. Pneumatic process in each cycle generally can be divided into four steps: compression wave introducing, compression wave reflecting, expansion wave introducing, expansion wave reflecting.

1. Compression wave introducing. During this phase, the tube is in the low pressure state, and the inner pressure is lower than the atmosphere pressure. the jet flows into the tube and the inner pressure rises. Compression wave generates near the mouth of tube and then walks into the tube until reaching the end of the tube. The pressure after the compression wave increases.

2. Compression wave reflecting. Compression wave is reflected on the end of the tube, and then compression wave walks out of the tube until reached the mouth of the tube. The pressure within rises and the velocity after compression wave drops. This phase is the preparation 
process of gas evacuation. The tube is filling and the pressure within reaches the top at the end of this phase. The first two phases are the filling process of the circle.

3. Expansion wave introducing. The jet initially flows out the tube. During this phase, the tube is in the high pressure state. Inner pressure is higher than the atmosphere pressure, so jet flows out of the tube and the pressure within decreases. Expansion wave generates near the mouth of tube and then walks into the tube until reaching the end of the tube. The pressure after the expansion wave decreases and the velocity after the expansion wave increases.

4. Expansion wave reflecting. Expansion wave is reflected on the end of the tube, and walks out of the tube until reaching the mouth of the tube. The pressure within decreases. The tube evacuates and the pressure within reaches the bottom at the end of this phase. The third and the fourth phase are the evacuation process of the circle. And until now a circle is fulfilled and the next circle begins.

\section{CONCLUSION}

Flow simulations have been performed in order to better understand the behavior of the PRT driven by annular jet. The simulation results show that it is feasible to use the PRT driven by annular jet to generate low frequency acoustic wave, and Pneumatic process in each cycle generally can be divided into four steps: compression wave introducing, Compression

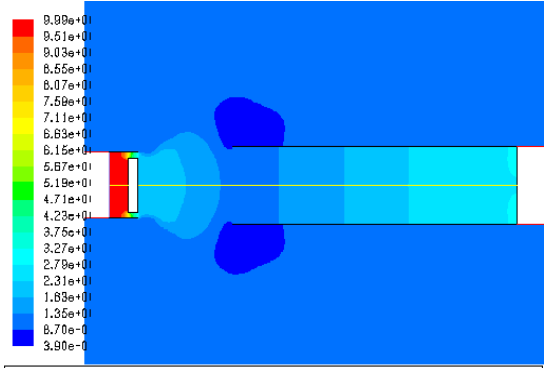

(a)

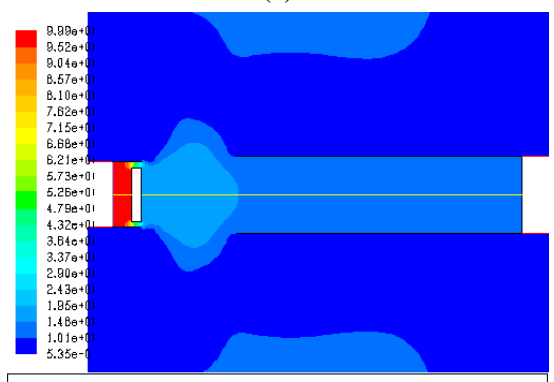

(b)

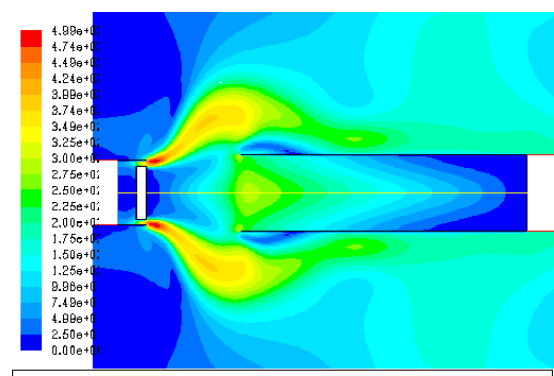

(a)

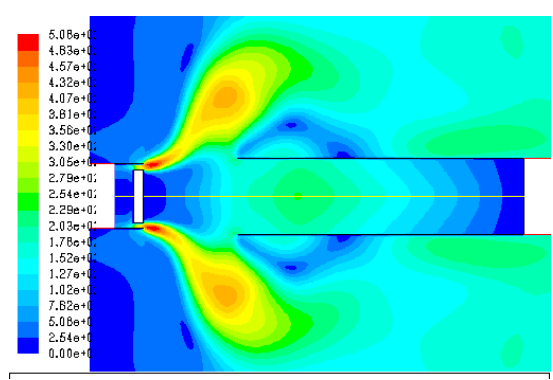

(b) wave reflecting, expansion wave introducing, expansion wave reflecting.

\section{ACKNOWLEDGMENT}

This research was supported by project SAST201314.

\section{REFERENCES}

[1] B. Brocher, Maresca. Fluid dynamics of the resonance tube. [J] AIAA, 1990, 43(2): 369-384.

[2] A. B. Cain, E. J. Kerschen. Simulation of acoustic characteristics and mechanisms of powered resonance tubes.[C]. AIAA/CEAS Aeroacoustics Conference, Breckenridge, CO, 2002-2400.

[3] Zhang Lijie. Ideal medium strong sound set of array technology research [D]. University of defense technology, 2008, 34-51.

[4] Hall IM, Berry CJ. On the heating effect in a resonance tube. J Aero Sci 1959;26(4):253.

[5] IwamotoJ.A study of flow oscillation and heating in a HartmannSprenger tube - a literature survey.In:Proceedings of the Institute of Mechanical

[6] A. B. Cain. Simulations of Helmholtz resonator powered resonance tubes at moderate pressure ratios[C]. AIAA Aerospace Sciences Meeting and Exhibit, Reno, Nevada, 2006-800.

[7] A. B. Cain. Simulation of powered resonance tubes: effects of pressure ratio and freestream flow[C]. AIAA Flow Control Conference, Saint Louis, 2002-2821.

[8] A. B. Cain. Simulation of powered resonance tubes: Helmholtz resonator geometries[C]. AIAA Flow Control Conference, Portland, Oregon, 2004-2690.

[9] E. J. Kerschen. Analytical modeling of helmholtz resonator based powered resonance tubes[C]. 2nd AIAA Flow Control Conference, Portland, Oregon, 2004-2691.

[10] A. Hamed, K. Das. Numerical simulation and parametric study of hartmann-sprenger tube based powered device[C]. 41st Aerospace Sciences Meeting and Exhibit, Reno, Nevada, 2003-550.

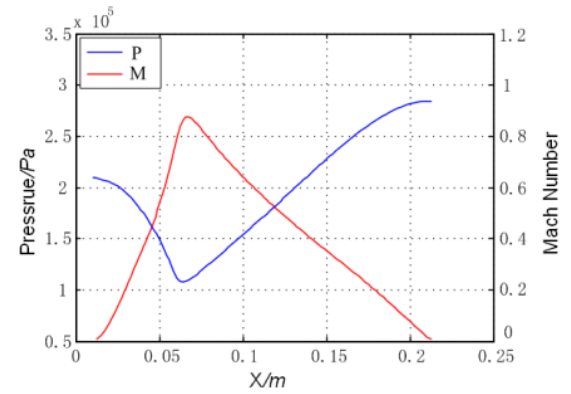

(a)

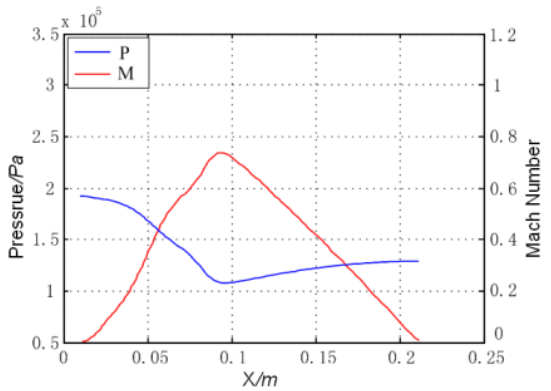

(b) 


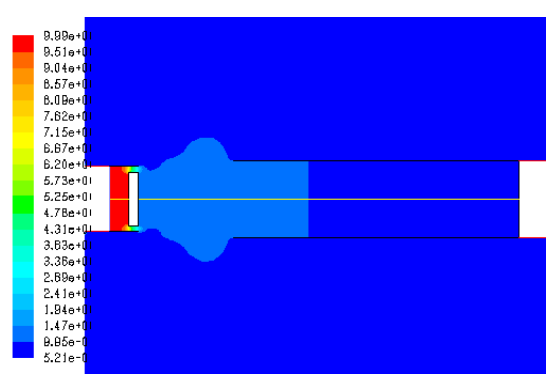

(c)

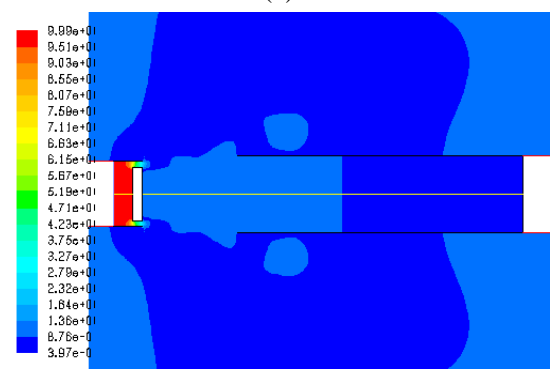

(d)

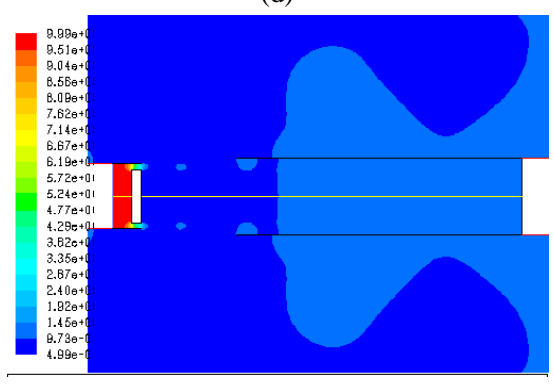

(e)

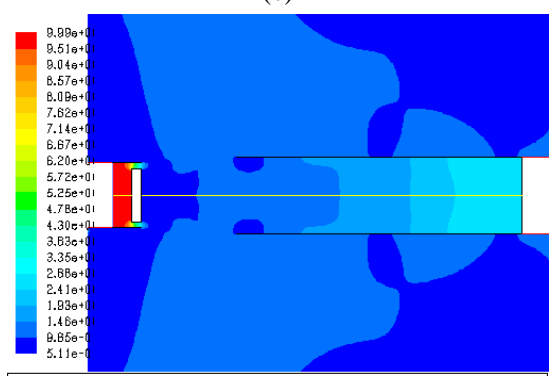

(f)

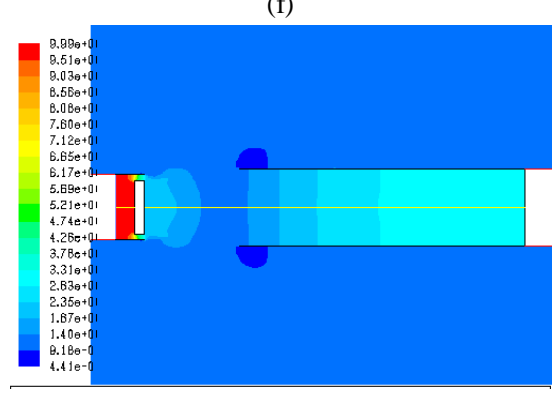

(g)

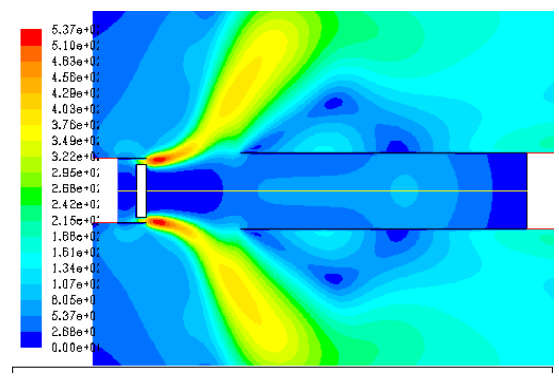

(c)

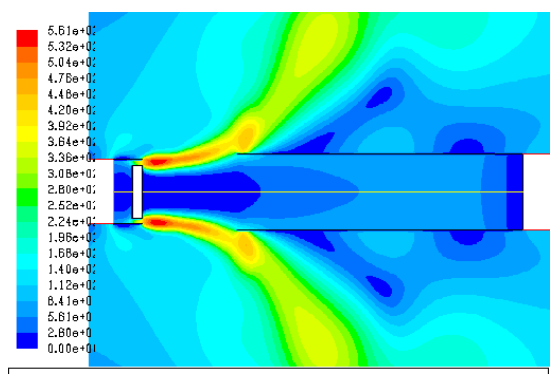

(d)

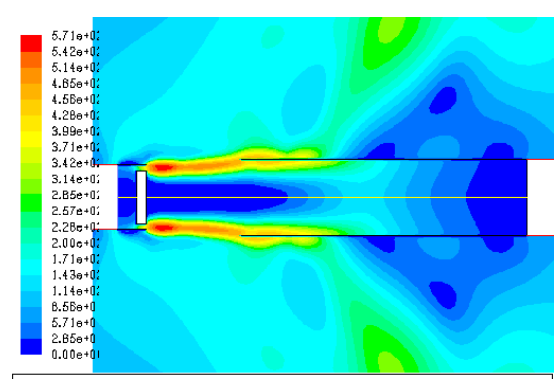

(e)

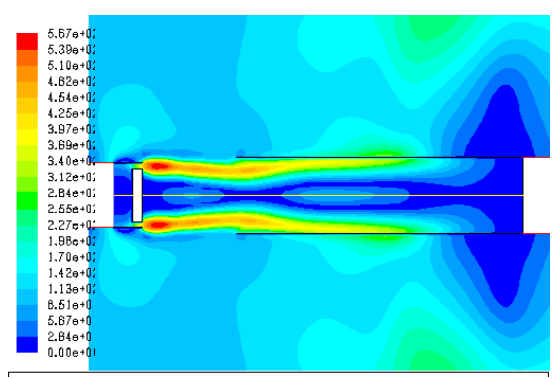

(f)

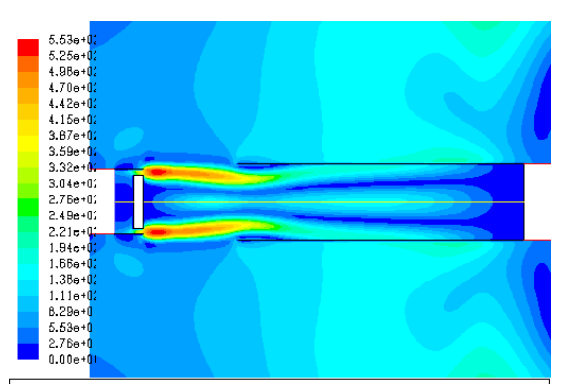

(g)

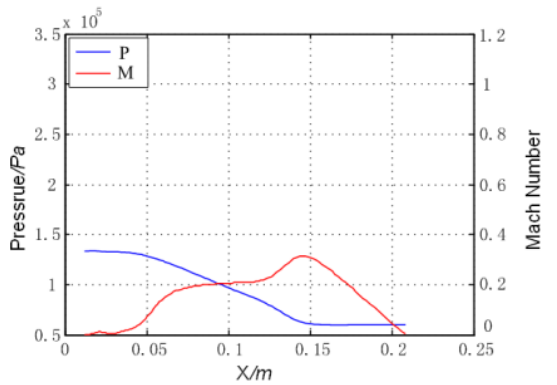

(c)

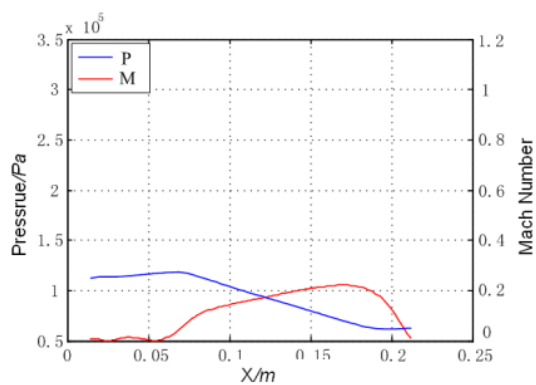

(d)

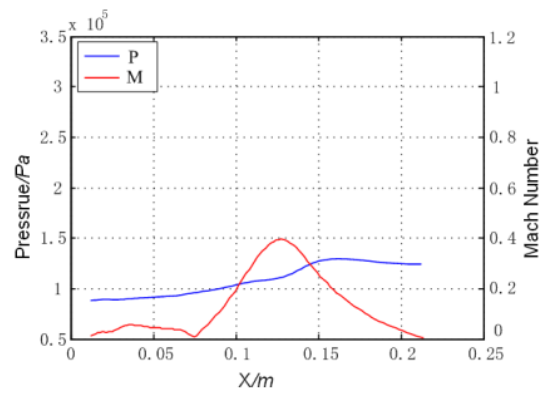

(e)

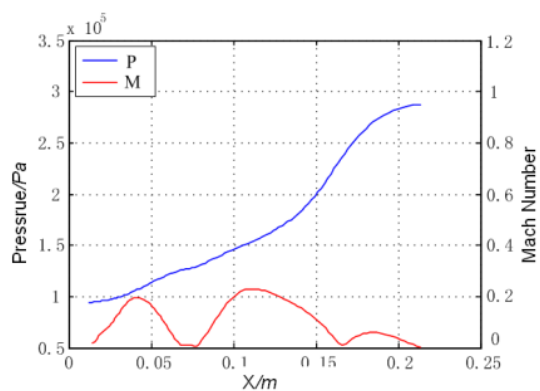

(f)

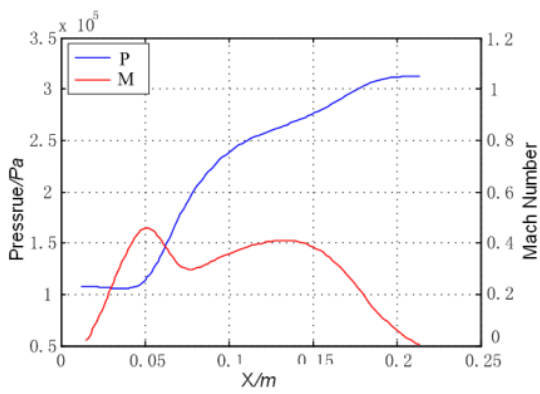

(g) 


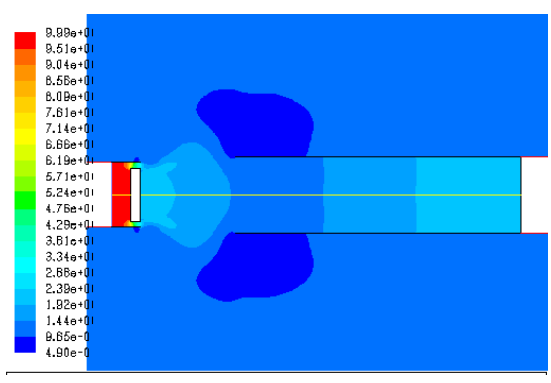

(h)

Figure 2. The static pressure distribution of PRT

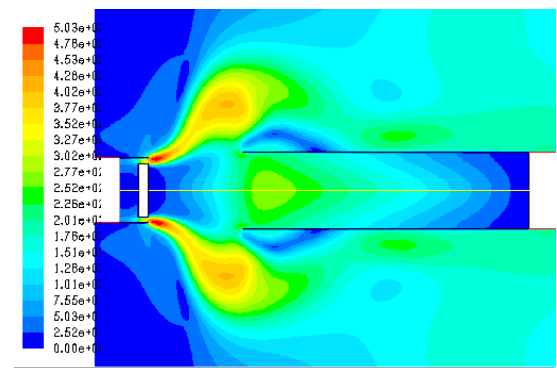

(h)

Figure 3. The velocity distribution of PRT

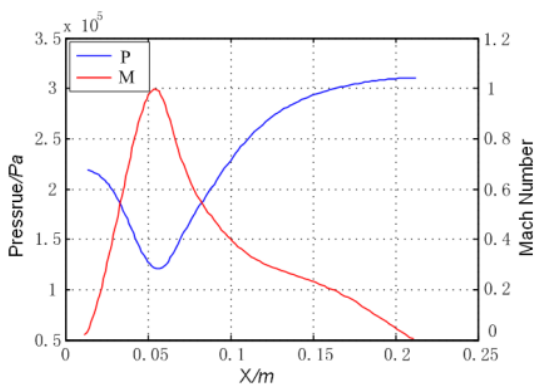

(h)

Figure 4. The static pressure and Mach number distribution on the axis 